\title{
Function of "Memes" in Adolescent Communication: A Theoretical Review
}

Rachel Myers ${ }^{1}$

Twenty years ago, distracted boyfriend, grumpy cat, and Kermit the Frog would have been three terms that had nothing in common. Today, they are instantly recognized by many as characters of some of the most popular memes. As technology rapidly progressed at the turn of the century, memes evolved to become a crucial aspect of internet culture. What remains to be seen, however, is how this influences communication, and whether or not there is a generational gap in understanding of this new way of communicating. This paper reviews the definition and a brief history of internet memes before discussing the common characteristics of memes. Research related to possible reasons for the popularity of memes, such as human preference for analogy and the inherent desire to belong to an ingroup, are also examined. Possible explanations for the generational divide in understanding and use of memes are considered. It is hypothesized that future experiments would reveal a discrepancy in fundamental understanding of memes across age groups.

\section{What is a meme?}

The term "meme" was first used to describe "a unit of cultural information spread by imitation" (Britannica, 2020). The original use of the word, coined by Richard Dawkins in The Selfish Gene (1976), encapsulated a broad range of ways that ideas are shared within a culture, from accents to clothing fads. The term was intended to relate cultural transmission to Dawkins' theory of of social evolution, in which memes were "genes," and natural selection of these genes results in some ideas surviving and others dying out (Isaacs, 2020).

In the context of internet and social media, a meme has been defined as "a virally transmitted image embellished with text, usually sharing pointed commentary on cultural symbols, social ideas, or current events," and usually addresses something that most people find relatable (Lifewire, 2020). Memes can be created in response to a current event or controversial topic, or can arise from different interpretations of an image or video (Lifewire, 2020). For the purposes of this paper, the modern definition of a meme that relates to internet culture serves as a starting point. It will also be used to consider further exploration of the psychology of internet culture.

${ }^{1}$ Princeton University 
What defines a meme and sets it apart from other aspects of internet culture is its propensity for mutation; many images and videos become "viral" online, but memes are unique in the way they are constantly being remade and reformed (McCulloch, 2019). McCulloch (2019) discusses the evolution of internet meme culture and how the internet evolved to make the production of new memes easier and accessible to everyone online. Much like the original definition of a meme, internet memes are changing and competing with one another constantly, with some fading out of relevance and others lasting for long periods of time.

Internet memes may have only existed for a short period of time, but the concept of a meme and how it relates to inherent human behaviors has always existed; for example, McCulloch (2019) mentions a popular form of graffiti from World War II that serves as an example of a "pre-internet meme," in that it was remade and reformed all over Europe. When the internet first became available, memes were only used by people who were familiar with the new technology and had the programming skills to create them, and anyone who was not familiar with these things was often left out of the joke (McCulloch, 2019). As the internet became more readily available and easier to use, memes grew in popularity and became more relatable.

McCulloch (2019) explains that when a meme format becomes extremely common, it may receive a name that all memes using that format fall under. One popular example of this is "Philosoraptor," which always features the same drawing of a velociraptor and text asking a question (see Figure 1). McCulloch (2019) goes so far as to categorize animal memes that emerged between 2008 to 2014 as "Advice Animal memes," which were always composed of a single character image and text about that

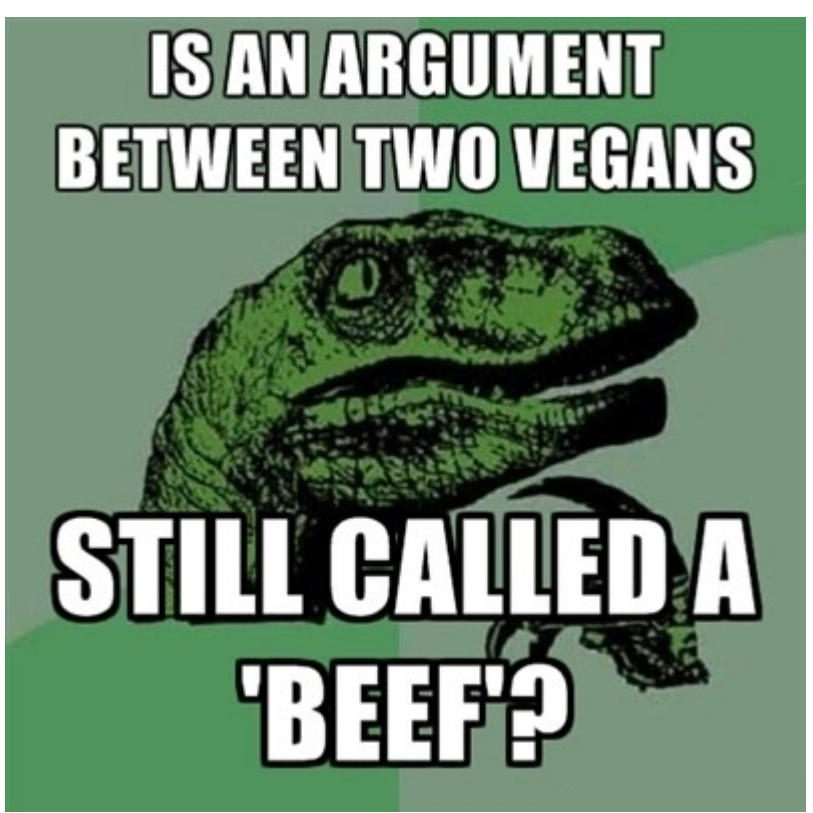
central character's thoughts or actions. Since then, internet meme formats have continued to evolve and spread, meme generation sites have become popular, and websites that compile and explain memes have appeared. Researchers and internet users alike began to realize that memes were always evolving, fading in and out of relevance, with the meme formats of 2016 having almost nothing in common with the formats of 2008 (2019). Meme culture is more than a fad, it is a living and growing form of culture and reflection of many important human characteristics, such as our capacity for analogical comparison and desire to belong.

Figure 1: This is an example of the Philosoraptor meme format. Often, these memes turn a play on words into a hypothetical question. 


\section{Analogy and Language}

Studies have shown that understanding analogies and relational language is not uniquely human; however, humans are superior at understanding analogy compared to apes (Haun \& Call, 2009). Christie et al. (2016) tested relational ability by hiding a treat in a three-tiered box, then asking them to guess where the treat would be in an identical box; if children and apes were using relational ability to select a box, they would perform better when the location of the treats were aligned (treat in top tier, treat is on the top tier) compared to when a rule was used to figure out where the treat was (treat on the top tier of the first box means the treat in the second box is on the middle tier). Researchers found that when simple treats were used, children and apes performed better in the aligned condition; however, when different objects were used to display the rule of the shifted condition, children performed better in the shifted condition, indicating that while children and apes have spatial relational ability, only children understand object relation (see Figure 2). This supports the evidence shown by Thompson et al. (1997) that some language-naive animals are capable of completing simple relational tasks. While animals may not require language to understand simple relations, children as young as four years old outperform other species in relational tasks and display better object focus than apes, a tendency that is likely attributed to language development (Christie et al., 2016; Christie \& Gentner, 2014).

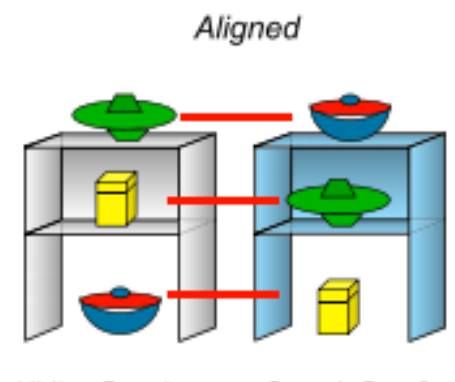

Hiding Box 1

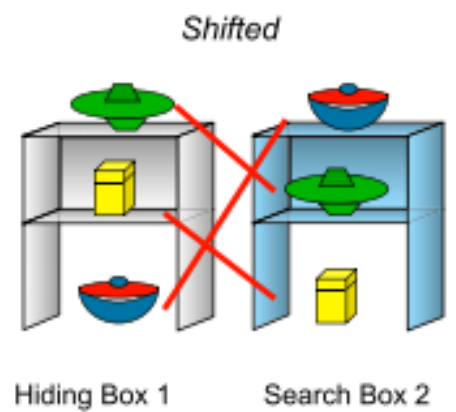

Hiding Box 1
Search Box 2

Figure 2: An image explaining the second trial of Christie et al. (2016), taken from the paper. Results showed that children were able to recognize the pattern in the shifted condition and complete the task, but apes were not.

Other studies have shown that language can be used to improve performance in relational tasks; for example, Ratterman and Gentner (1998) used words like "mommy" and "baby" to convey changes in size between objects; results showed that children in these groups were significantly better at completing relational tasks compared to children who were given no labels (1998). Another study showed that three-year-old children can improve relational abilities by simply being exposed to relational object labels like "same" and "different" (Christie \& Gentner, 2013). These studies suggest that human capability for language makes us better equipped to understand and communicate analogies. 
As McCulloch (2019) discusses, a meme format becomes humorous once you understand the types of analogies the format uses and appreciate the creative comparisons being made as the meme continues to be recreated. Often, understanding a new meme requires seeing several versions of the same format; once you learn the "rule" of the new meme, you can appreciate the humor behind it and contribute to recreating it. This makes meme culture linguistically significant and allows for old meme formats to continue to evolve and new meme formats to take over the internet: as long as there are comparisons to be made, memes can be made, and as long as you understand the basic process of "learning" a meme, you are capable of enjoying any "fresh meme" you happen across.

\section{Social Impact of Memes: the Psychology of In-groups}

Internet memes are extremely versatile, and almost any format can be used to relate to almost any topic; what makes memes unique, is that understanding them means you identify with internet culture, as well as the subgroup (parents, dog owners, or college students, etc.) that the meme relates to (McCulloch, 2019). McCulloch (2019) suggests that memes derive their popularity from an appeal to the human desire to fit in, to belong to a group of people; "getting" a meme means you fit in.

This idea aligns with research on human behavior. Humans tend to prefer to be part of an "ingroup," or a subset of people that they feel they belong to; in addition, ingroups often adopt certain symbols or behaviors that distinguish them from "out" groups (Brewer, 1999). Humans desire a sense of belonging and tend to separate themselves and those they identify with from the rest of the world. Memes serve as a way to do this virtually.

For adolescents, a sense of belonging is highly desired. In a review of studies on the influence of adolescents on language, linguist Penelope Eckert (2003) suggests that adolescents are responsible for many of the major shifts in language and tend to decide what is popular and what isn't. Eckert (2003) focuses on the prevalence of ingroups in adolescents created through the use of variation in language. For one, adolescents use labeling as social control and to draw lines between themselves and other groups they do not wish to be associated with, such as athletic students being referred to as "jocks" and high achieving students being referred to as "nerds" (Eckert, 2003). Labels can also be used to exclude people who do not know the meaning behind the label, separating them from the members of an ingroup, such as when a group of friends give someone a nickname that has a backstory only they know (Eckert, 2003). Slang words are also popular among adolescents for the same reason, allowing them to identify with certain groups through the use of certain terms; for example, white students may adapt aspects of African American English to make themselves appear cooler or tougher (Eckert, 2003). Internet memes serve the same purpose, with adolescents being able to create and share memes that relate them to their ingroup and show that they are up to date with internet culture. Memes can be used to express who you are and how you relate to others.

\section{Further Exploration}

It is likely that internet memes are the new format for creating ingroups and communication in adolescents and young adults; if Eckert (2003) was correct in her 
theory that adolescents control linguistic and cultural shifts, then memes can offer insight. It is also necessary to understand whether or why memes are not a popular form in older groups of people who use the internet. If memes are just analogies, and all humans are capable of understanding analogies, then (controlling for familiarity with the topic of comparison) all age groups should be capable of understanding and appreciating memes. If it is a result of being raised using the internet versus learning to use the internet as an adult, then separating memes from using the internet (i.e., printing multiple examples of a meme format on paper) should allow any age group to understand a meme; however, I hypothesize that this would not be the case. There may be some linguistic or psychological experiences with memes or with adolescent culture that older generations lack. It is possible that, like language learning, learning the rules of memes becomes more difficult with age.

Lack of interest or understanding of memes in older adults could be related to the use of images to convey the analogy. Languages spoken today do not use images, meaning mastery of a language, like English, would not guarantee an understanding of more symbolic forms of communication. Again, this would imply a relationship between memes and language learning, as younger people may be more capable of learning to use symbolism as a means of conveying information. Memes often only use text to list the things being compared, and it is the image that is used to convey the relationship or commonality between them (see Figure 4). The context of where the meme came from does not seem to be necessary for understanding the meme (teenagers who do not watch Spongebob are still capable of enjoying the memes that use images from the show), but it could be considered a possibility. If it is the case that images used in memes confuse older age groups, then it is possible an experiment could be conducted comparing age groups and their ability to understand and utilize an unfamiliar symbolic language.

It is possible that younger age groups would show a greater aptitude for understanding images and symbols. This could indicate a shift in language development of children in developed countries from words alone to more pictorial forms of communication. Memes represent an important shift in communication, language, and culture, and can offer insight on adolescent socialization as well as shifts in how society interacts. Understanding what divides age groups in their appreciation of meme culture can shed light on how language and communication are changing and how we learn to understand those changes. 

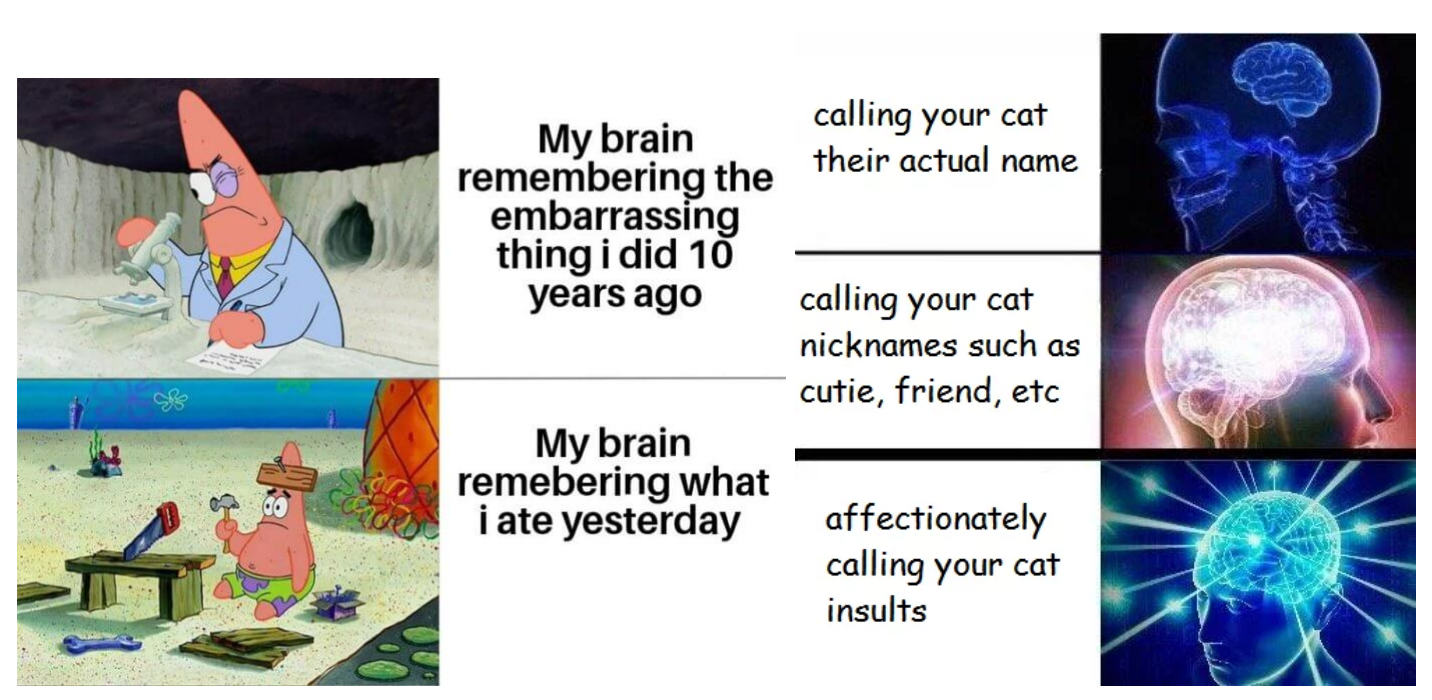

Figure 4: These are examples of the tendency of memes to use images to convey analogy and text strictly to list items being compared.
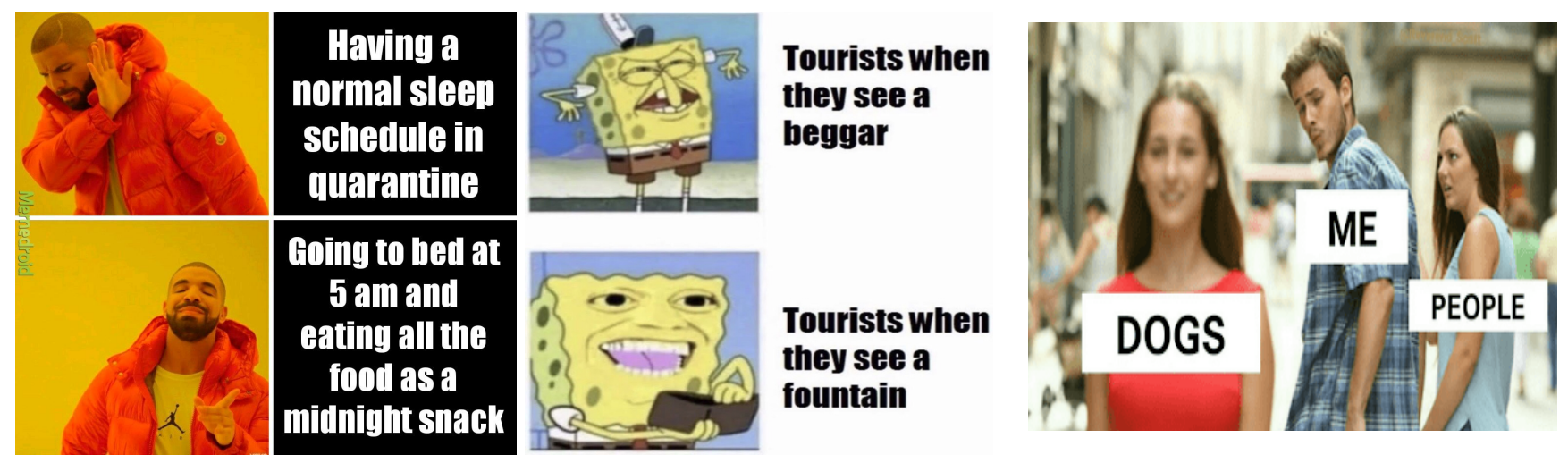

Figure 5: These meme formats use the same type of analogy, with reactions of characters indicating one item described is preferred over the other.

\section{Gardener Comments}

\section{Anonymous1:}

This article discusses a contemporary form of communication through two theoretical frames, namely linguistic processing and the psychology of in-groups. The central idea is novel, interesting and, as argued by the author, important. There are also some speculations put forward on how the ideas discussed can be empirically explored; for instance, testing meme comprehension and controlling for "familiarity with the topic of comparison" and "printing multiple examples of a meme format on paper" to take into account relative internet content fluency. 
Therefore, whilst no specific experiment or study is proposed, it can be argued that this article has great value: through its potential to spark further and more particular research, premised on these initial ideas. Such ability is how this journal can be unique in the ideas it inspires.

\section{Roger's Bacon:}

While it is largely a review of previous work on memes and adolescent communication, I find it to be a useful introduction to these subjects and some of the ideas in the "further explorations" section could stimulate future research. With some reservations (see below), I recommend this article for publication.

I find the notion that "adolescents are responsible for many of the major shifts in language" to be an interesting one and I wonder to what degree the internet has and will shift our communication style because of the fact that it gives a larger voice for teens and young adults to contribute to the greater cultural conversation. Is there a sense in which our culture and language is shifting towards a cognitive style more characteristic of teens? Our current technological environment has at the same time reduced the voice of older adults in the wider culture as they are less likely to participate in the internet/social media and their experiences are less relevant to the youth than they have been in previous generations (e.g. many older adults have no experience with online dating and therefore cannot relate to an important aspect of modern culture). All of this would suggest that our culture and language is becoming "younger". Anecdotally, this seems very reasonable, but I would be interested in a more rigorous test of the hypothesis. Some might have the intuition that a more "teenage" human culture would be a bad thing, however I contend that it simply represents a tradeoff (e.g. more creativity, less stability) and that we would be wise to create systems that help accentuate the strengths of this cultural shift and mitigate the weaknesses (easier said than done of course).

I'm not sure about the relevancy of the primate example. It should be fairly obvious that humans have better relational ability than other primates and this doesn't really seem to bear on the difference between teens and older adults which is more central to the article. Is there research on differing relational ability, language usage, or metaphor usage in adults vs. teens? This may be good to cite.

The "further" explorations section is good, but I feel like it would be improved if there were more specific suggestions (hypotheses, experiments).

\section{R. Sal Reyes:}

I can imagine deeper explorations of specific kinds of meme usage generating novel behavioral insights-e.g., determining how or if a specific type of content reliably increases a meme's viral success in different populations or cultures, or exploring the specific role of memes in helping to build \& sustain today's fringe political movements. However, this paper's primary premises regarding memes' centrality in today's adolescent culture, memes' use of language and imagery to generate analogies, and memes' helping to confer in-group status seem too broad ( $\&$ too face-value as 
observations) to generate any genuinely novel insights about memes' roles among youth or how they work as communications \& bonding tools (beyond explorations of those topics that are already occurring).

In addition, the hypothesis driving the paper-that older adults (who are ambiguously-defined) are less capable of understanding memes for some reason beyond a simple lack of exposure to their usage-is not supported by sufficiently specific reasoning or evidence. And, in fact, the example noted above about memes' usage in fringe political groups suggests that there is ample evidence of memes being effective communication \& bonding tools in groups with a wide range of individuals of different ages. Speculations about matters like "learning the rules of memes becomes more difficult with age" or "younger age groups show a greater aptitude for understanding images or symbols" are not grounded in any specific observations or reasoning here, and thus feel like shots in the dark as opposed to solid hypotheses that could generate useful results.

\section{Dudley Dumpling:}

There were definitely aspects of your paper that I found interesting, and you provoked some ideas that I hadn't really ever considered (such as the natural selection and survival of ideas)

I think that based on SoS criteria, your paper could be published. Personally, I did not find your thesis particularly novel or intriguing enough, and I don't necessarily agree with it. I can however see how someone could disagree with me.

I do not think that older generations are any less capable of understanding or getting memes and meme-related jokes on the internet. I think many adults do enjoy and share memes (like our parents on facebook and increasingly instagram), and I think the oldest generation simply doesn't care, or are left out of the conversation.

I think the paper was written well enough to be published, but because of my feelings toward your thesis I don't think it is quite enough.

\section{Alex Gillespie:}

The idea of memes as a distinctive and new symbolic form based on a mapping is interesting and worth developing and publishing. I'm less convinced by the argument around age simply because I know many older people who enjoy memes. To continue the argument about age, I recommend more evidence about age discrepancies and some critical self-reflection about alternative sources of any age differences. For example, as with many new modes of communication, it may be a cohort effect, and in 40 years there will be no age differences. The argument could also be strengthened if there were any other modes of communication or symbolic forms that show age effects.

\section{Sergey Samsonau:}

The manuscript does not explain why existing approaches describing language evolution are not sufficient to describe evolution of memes. 


\section{Sander Van de Cruys:}

I found this to be a very nice and concise overview of the work on the psychology of memes. However, the central thesis, namely that memes are better processed/understood by younger people, is insufficiently substantiated. Specifically, if for example analogical reasoning is assumed to be not just present but better in children/adolescents, this should have already been empirically established in the literature (not that I know of, but I'm not an expert here). If it is just a form of vernacular used to socially distinguish oneself as part of the group, there is no essential difference in processing between adults and adolescents (it's just a matter of learning the specific language). So the authors did not convince me that the clearly higher prevalence of memes among youth implies different processing (or improved processing fluency per se for memes as a category of stimuli), rather than just different levels of exposure to memes and their specific regularities. I did not reject the paper, because I think it has potential and is interesting, but l'd highly recommend to try to strengthen or elaborate the main thesis about the link between memes and their better understanding (so not just increased use/exposure) in young folks if one wants to keep it as the main focus. With regard to the social dimension of memes, l'd highly recommend reading the chapter "A populism of lulz" by Wagner \& Schwarzenegger ( 2020).

\section{Anonymous2:}

1) It may be worth mentioning that meme creation doesn't have to be a means of ingroup affiliation but rather happen for its own sake, as an intellectual/humorous exercise. 2) Do memes necessarily involve images? At least on twitter there are plenty of circulating memes that use nothing but words. 3) It might also be interesting to examine what analogies of memes existed in pre-internet times (though that's admittedly a subject of a whole other paper).

\section{Judah:}

I'm not entirely sure this would be considered "serious science" but it does do a good job of making explicit some of the primary functions of memes in the context of the current internet. If it so happens that this sort of analysis hasn't already been done, then yes, this ought to be published.

\section{Richard Roos}

Author misses that memes also convey specific feelings and emotions. At least for some people. Some images are easier associated with emotions than words/texts. E.g. "Sad Keanu" meme actively elicits a feeling of sadness or loss. I think this ability to trigger emotions is one of the causes of meme popularity as a group-building device. 


\section{$\underline{\text { References }}$}

1. Brewer, M. (1999). The psychology of prejudice: in group love or outgroup hate? Journal of Social Issues, 55(3), 429-444.

2. Christie, S., Gentner, D. (2014). Language helps children succeed in classic analogy task.

Cognitive Science, 38(2), 383-397. https://doi.org/10.1111/cogs.12099.

3. Christie, S., Gentner, D., Call, J., \& Haun, D. B. M. (2016). Sensitivity to relational similarity

and object similarity in apes and children. Current Biology, 26(4), 531-535.

4. Eckert, P. (2003). Language And Adolescent Peer Groups. Journal of Language and Social Psychology, 22(1), 112-118.

https://doi.org/10.1177/0261927X02250063

5. Gil, P. (2020, June 22). What is a Meme? Lifewire.

https://www.lifewire.com/what-is-a-meme-2483702

6. Haun, D, Call, J. (2009). Great apes' capacities to recognize relational similarity. Cognition, 110(2), 147-159. https://doi.org/10.1016/j.cognition.2008.10.012.

7. Isaacs, D. (2020). Memes, Journal of Paediatrics and Child Health, 56(4), (497-498), (2020). McCulloch, G. (2019). Because Internet: Understanding the New Rules of Language. Riverhead Books.

8. Thompson, R. K. R., Oden, D. L., \& Boysen, S. T. (1997). Language-naive chimpanzees (Pan troglodytes) judge relations between relations in a conceptual matching-to-sample task. Journal of Experimental Psychology: Animal Behavior Processes, 23(1), 31-43. https://doi.org/10.1037/0097-7403.23.1.31

9. Rattermann, M. J ., \& Gentner, D . (1998). The effect of language on similarity : The use of relational labels improves young children's performance in a mapping task . In K. Holyoak, D . Gentner, \& - B. Kokinov (Eds.), Advances in analogy research : Integration of theory and data from the cognitive, computational, and neural sciences, 274-282. Sophia: New Bulgarian University. 